\title{
Prevalência de transtornos mentais comuns em jovens brasileiros recém-incorporados ao Serviço Militar Obrigatório e fatores associados
}

\author{
Prevalence of common mental disorders in recently-drafted young \\ Brazilians to mandatory military service and associated factors
}

Lilian Cristina Xavier Martins ${ }^{1}$

Leandro Kuhn ${ }^{1}$

\footnotetext{
${ }^{1}$ Escola de Educação Física do Exército, Diretoria de Pesquisa e Estudos de Pessoal, Ministério da Defesa, Exército Brasileiro. Av. João Luis Alves s/n, Urca. 22291-090 Rio de Janeiro RJ.

lilitinac@yahoo.com.br
}

\begin{abstract}
The mental health of the military enlisted in the Armed Forces is an as yet area little explored of research in Brazil. This study aimed to investigate the prevalence of common mental disorders (CMD) and associated factors among recently-drafted young Brazilians to mandatory military service: the recruits. A total of 78 young adults participated in the study. The incidence of CMD (dependent variable) were evaluated by the 12-item General Health Questionnaire (GHQ12). The tests ?' (chi-squared) and logistic regression were used to estimate the associations between CMD and independent variables. The results showed a prevalence of CMD de $43.6 \%$ (CI 95\% 33.14 to 54.64). The CMD prevalence ratios were from 4 to 5 times higher among those who reported sleep disturbances, which was the only factor associated with the occurrence of CMD. These results seem to indicate that the situations experienced by the recruits in their work environment may be causally associated with CMD. Further studies are needed, with longitudinal design, for this effect to be confirmed.
\end{abstract}

Key words Sleep disturbances, Common mental disorders, Soldiers, Mental health
Resumo A saúde mental de militares das Forças Armadas é uma área de investigação ainda pouco explorada no Brasil. O presente estudo teve por objetivo estimar a prevalência de transtornos mentais comuns (TMC) e identificar os fatores a estes associados em jovens brasileiros recém-incorporados ao serviço militar obrigatório: os recrutas. A população de estudo foi composta de 78 jovens adultos do sexo masculino. A ocorrência de TMC (variável dependente) foi avaliada por meio do General Health Questionnaire, versão 12 itens (GHQ-12). Para estimar as associações entre TMC e as variáveis independentes foram utilizados o teste $\chi^{2}$ (Qui-quadrado)e a regressão logística. Os resultados mostraram uma prevalência de TMC de 43,6\% (IC 95\% 33,14 - 54,64). As razões de prevalência de TMC mostraram-se entre 4 e 5 vezes maiores entre os que apresentavam distúrbios do sono, sendo que estes se mostraram como único fator associado a TMC. Estes resultados parecem indicar que as situações vivenciadas pelos recrutas em seu ambiente de trabalho podem estar associadas com TMC de forma causal. Outros estudos, com desenho longitudinal, são necessários para que tal efeito seja confirmado.

Palavras-chave Distúrbios do sono, Transtornos mentais comuns, Militares, Saúde mental 


\section{Introdução}

As saúdes mental, física e social são dimensões estreitamente entrelaçadas e profundamente interdependentes da vida humana ${ }^{1}$, sendo a primeira indispensável para o bem-estar geral dos indivíduos. Não obstante, a frequência de transtornos mentais no mundo é bastante elevada. Segundo a Organização Mundial da Saúde $(\mathrm{OMS})^{1}$ a depressão grave é a principal causa de incapacidade, ocupando a quarta posição entre as dez principais causas de outras patologias. Os distúrbios não psicóticos, principalmente depressão e ansiedade, ocorreram em cerca de $90 \%$ dos indivíduos estudados com problemas psiquiátricos ao redor do mundo ${ }^{2}$.

O desenvolvimento de doenças físicas e mentais é influenciado por componentes biológicos, psicológicos e sociais. Nessa perspectiva, a literatura mostra que existem fatores que aumentam a probabilidade de ocorrência de transtornos mentais como: pobreza, sexo, idade, ocorrência de conflitos e catástrofes, doenças físicas graves e ambientes familiar e social ${ }^{1,3}$, situação conjugal, desemprego $^{3}$, aptidão cardiorrespiratória ${ }^{4}$, estado nutricional ${ }^{5}$ e distúrbios do sono ${ }^{6}$. A frequência de transtornos mentais é alta na população em geral e, por vezes, apresentam-se como comorbidades, como é o caso de ansiedade combinada com perturbações depressivas ${ }^{1}$. Os sintomas de depressão e de ansiedade são muito comuns na população adulta, sendo diagnosticados em um terço ou mais das pessoas que procuram atendimento em serviços primários de saúde ${ }^{3}$, entretanto, estudos apontam que, mesmo em países desenvolvidos, somente $40 \%$ dos indivíduos que tiveram conclusos diagnósticos para transtornos de ansiedade ou de depressão recebem o respectivo tratamento ${ }^{7}$. Com os avanços no conhecimento científico na área da saúde mental, foi estabelecido o termo "transtornos mentais comuns" (TMC) que se refere a uma situação na qual o indivíduo, que não preenche os critérios formais para diagnósticos de depressão e/ou ansiedade, apresenta sintomas tão proeminentes que trazem uma incapacidade funcional comparável ou até pior do que quadros crônicos já bem estabelecidos ${ }^{8}$, sendo que TMC estão entre as principais causas de incapacidade 9 .

Em países desenvolvidos, na população em geral, as prevalências de TMC variam de 7 a $30 \%{ }^{10}$. No Brasil, em Pernambuco, essa prevalência, na população em geral, foi de $35 \%{ }^{11} \mathrm{e}$, em Pelotas, foi de $28 \%$ (homens e mulheres), sendo que somente entre os homens foi de $23,5 \%{ }^{12}$.
Considerando populações de militares das Forças Armadas, no Reino Unido, estudo conduzido em 4.500 militares, exibiu uma prevalência de TMC de $20 \%{ }^{13}$. Nos Estados Unidos, pesquisa realizada com 12.756 participantes, das três Forças (terrestre, marítima e aérea), encontrou uma prevalência de $27,9 \%$ de sintomas depressivos ${ }^{14}$. No Brasil, de modo geral, há escassez de pesquisa na área da saúde nas Forças Armadas. Especificamente, em saúde mental, foi identificado somente um estudo, focalizando-a em relação a transtorno de estresse pós-traumático, referindo-se à participação de militares brasileiros em conflitos de guerra. Essa pesquisa foi realizada por ocasião do retorno de um dos contingentes, enviados pelo Brasil ${ }^{15}$, para atuar em missão de paz promovida pela Organização das Nações Unidas (ONU).

Anualmente, cerca de 70 mil jovens brasileiros são incorporados às fileiras de soldados nas Forças Armadas para prestar o serviço militar obrigatório ao País ${ }^{16}$. A relevância do presente estudo fica ressaltada face à lacuna na literatura, pois, no Brasil, não foi identificado nenhum estudo sobre saúde mental de jovens recrutas, nem sobre a prevalência de transtornos mentais comuns entre militares.

O presente estudo teve por objetivo estimar a prevalência de transtornos mentais comuns (TMC) e identificar os fatores a estes associados, em jovens brasileiros recém-incorporados ao serviço militar obrigatório: os recrutas.

\section{Métodos}

\section{Desenho e população de estudo}

Este estudo é de desenho seccional em que se buscou obter uma amostra representativa de todos os soldados que foram incorporados ao Exército Brasileiro (EB) no estado do Rio de Janeiro em 2010. A população de estudo foi delineada como o censo, ou seja, todos os recrutas designados para servir em uma Diretoria do EB. O critério de inclusão foi ter sido incorporado ao EB, como soldado no serviço militar obrigatório, no ano de 2010. Seriam excluídos aqueles que não tivessem realizado o primeiro Teste de Aptidão Física (TAF) de 2010 ou que estivessem em missão de longa duração, que impossibilitasse a participação na pesquisa. Dos 122 recrutas, um não havia realizado o primeiro TAF e 43 estavam em missão de longa duração. O número total de elegíveis foi 78. A coleta de dados se deu durante o mês de março de 2010. 
onimato e o necessário voluntariado para participação. Todos os que concordaram em participar assinaram o Termo de Consentimento Livre e Esclarecido e foram observados todos os princípios éticos que regem a pesquisa científica envolvendo seres humanos.

\section{Mensurações}

\section{Transtornos mentais comuns}

Para se avaliar a variável dependente, que foi TMC, utilizou-se a versão com 12 questões do General Health Questionnaire versão 12 itens (GHQ-12) $)^{17}$. O GHQ-12 foi traduzido e validado no Brasil ${ }^{18,19}$. O GHQ-12 é um instrumento amplamente utilizado em estudos epidemiológicos para rastreamento de TMC. Compõe-se de 12 perguntas, com uma escala de quatro pontos para cada resposta, que se referem ao quanto dos sintomas de ansiedade e/ou depressão a pessoa tem experimentado. Nas questões que apresentam diretamente um problema de saúde mental (como as perguntas sobre: Perder muito sono por preocupação; Sentir-se constantemente nervoso(a) e tenso(a; Sentir que está difícil de superar suas dificuldades; Sentir-se triste e deprimido; Perder a confiança em você mesmo; Achar que é uma pessoa sem valor) apresentam respostas que variam de 1 ( De jeito nenhum) a 4 (Muito mais do que o costume). Já as questões que demonstram uma atitude mental positiva (como as perguntas sobre: Ter sido capaz de manter a atenção nas coisas que está fazendo; Sentir que é útil na maioria das coisas do seu dia-a-dia; Ser capaz de enfrentar seus problemas; Sentir-se capaz de tomar decisões; Sentir-se razoavelmente feliz de modo geral?; Ter satisfação nas suas atividades do dia-a-dia?) as respostas foram de 1 (Mais do que o costume) a 4 (Muito menos que de costume). Em todas as perguntas, respostas mais próximas de 1 indicam boa saúde mental; mais próximas de 4 apontam problemas de saúde mental. O ponto de corte, para identificar os casos de TMC, foi utilizado considerando cada item como ausente ou presente ( 0 e 1 ) - de acordo com o método do General Health Questionnaire ${ }^{17}$. Para tal, uma resposta 1 ou 2 foi considerada como ausente (0), enquanto uma resposta 3 ou 4 foi conside- rada como presente (1). Aqueles que foram positivos para 3 itens ou mais do GHQ-12 foram classificados como casos de TMC.

\section{Variáveis independentes}

Os fatores examinados quanto à associação com TMC foram as características: socioeconômicas e demográficas (escolaridade, renda familiar per capita, e situação conjugal), estilo de vida (fumo e consumo de álcool), estado nutricional (avaliado por meio do Índice de Massa Corporal: IMC) e aptidão cardiorrespiratória. Renda foi categorizada em salários mínimos. As variáveis sobre estilo de vida referiram-se ao hábito atual de fumar e ao consumo de qualquer quantidade de álcool nas duas últimas duas semanas. O IMC foi acessado com as medidas de peso e altura, coletadas pela Seção de Saúde no momento da incorporação e foi adotada a classificação do Ministério da Saúde ${ }^{20}$ : IMC $<18,5 \mathrm{Kg}$ "Abaixo do peso"; $>18,5$ e $<25$ "Peso Normal"; $>25$ e $<30$ "Sobrepeso" e $>30$ "Obesidade". A aptidão cardiorrespiratória foi analisada utilizando-se os valores individuais de $\mathrm{VO}_{2}$ max, expressos em $\mathrm{mlO}_{2} /$ $\mathrm{kg} / \mathrm{min}$. $\mathrm{O} \mathrm{VO}_{2} \max$ foi calculado utilizando-se o resultado do Teste de Cooper ${ }^{21-23}$, realizado pelos recrutas como parte integrante do primeiro TAF (realizado em março de 2010). Esta variável foi categorizada da seguinte forma ${ }^{23}$ : em pessoas entre 13 e 19 anos de idade, para um $\mathrm{VO}_{2 \max }$ $(\mathrm{mlO} / \mathrm{kg} / \mathrm{min})$, considera-se a aptidão cardiorrespiratória: até 35,0 "Muito Fraca"; de 35,1 a 38,3 "Fraca"; de 38,4 a 45,1 "Regular"; de 45,2 a 50,9 "Boa"; de 51,0 a 55,9 "Excelente" e acima de 56,0 "Superior". Distúrbios do sono ${ }^{6}$ foram avaliados por meio de duas questões, a respeito do período de duas últimas semanas: 1) Com que frequência você teve dificuldade para pegar no sono?; e 2) Com que frequência você acordou durante o sono e teve dificuldade para dormir de novo?. Com as seguintes opções de resposta: 1) Sempre; 2) Quase sempre; 3) Às vezes; 4) Raramente; e 5) Nunca. Posteriormente, estas foram dicotomizadas como se segue: as respostas "Raramente" e "Nunca" foram classificadas (codificadas) como ausente (0) e as respostas "Sempre", "Quase sempre" e "Às vezes", como presente (1).

\section{Análise Estatística}

As análises estatísticas foram iniciadas pela exploratória e pelas descritivas de cada uma das variáveis investigadas.

Para avaliar a associação das variáveis independentes com TMC, foi realizada a análise bi- 
variada por meio do teste $\chi 2$ (Qui-quadrado) e regressão logística, via modelos lineares generalizados. As variáveis que mostraram significância estatística $(\mathrm{p}<0,05)$ entraram na modelagem multivariada. $\mathrm{Na}$ análise multivariada, utilizouse regressão logística múltipla. A modelagem partiu de um modelo completo e com o procedimento de eliminação das variáveis que perderam sua significância estatística (backward) para se delinear o modelo final. As razões de prevalência e os respectivos intervalos a $95 \%$ de confiança (IC95\%) foram calculados.

Dados ausentes foram excluídos das análises e o nível de confiança estabelecido em todas as análises foi de $95 \%$. Os dados foram digitados no MSAccess 2000 e as análises estatísticas realizadas na Plataforma $\mathrm{R}^{24}$.

\section{Resultados}

A média de idade dos participantes foi de 18,97 $( \pm 0,9)$, sendo todos do sexo masculino, exercendo funções semelhantes inerentes ao serviço militar obrigatório. A prevalência de TMC foi de 43,6\% (IC95\% 33,14 - 54,64). A maioria apresentou renda per capita familiar de até 2 salários mínimos $(85,9 \%)$, era solteira $(94,9 \%)$, tinha escolaridade até o $2^{\circ}$ grau - conforme se exibe na Tabela 1. A maioria apresentava estado nutricional adequado (76,9\%), exibindo "Peso normal"20. A média de $\mathrm{VO}_{2 \max }$ de 53,96 $( \pm 7,65) \mathrm{mlO} 2 / \mathrm{kg} /$ min, sendo que $92,3 \%$ apresentaram aptidão cardiorrespiratória variando de "Boa" a "Superior" ${ }^{23}$. Ainda na Tabela 1, exibem-se os resultados da análise bivariada e observa-se que somente os distúrbios do sono apresentaram-se associados à TMC $(p<0,05)$. O estado nutricional apresentou um $p$-valor limítrofe $(0,067)$.

A Tabela 2 apresenta os resultados em razões de prevalências de TMC segundo distúrbios do sono. Aqueles que apresentavam dificuldade para dormir $(\mathrm{RP}=4,17 ; 1,18-14,77)$ e os que acordavam no meio da noite e tinham dificuldade para voltar a dormir $(\mathrm{RP}=5,69 ; 1,43$ - 22,75) exibiam prevalências muito maiores de TMC comparando com os que não apresentavam distúrbios do sono.

\section{Discussão}

O principal achado do presente estudo foi que, na população avaliada, os distúrbios do sono "ter dificuldade para adormecer" e "acordar no meio da noite e ter dificuldade para voltar a dormir" constituíram-se como únicos fatores associados à ocorrência de TMC. A população de estudo foi composta por jovens adultos, saudáveis, todos com cerca de 19 anos de idade, sem grandes disparidades de renda e escolaridade, e de aptidão cardiorrespiratória. Tal homogeneidade de carac-

Tabela 1. Características da população de estudo $(\mathrm{N}=78), 2010$

\begin{tabular}{|c|c|c|c|}
\hline Características & $n$ & $\%$ & $p$ \\
\hline Escolaridade & & & 0,724 \\
\hline Superior & 2 & $(2,6)$ & \\
\hline Médio & 67 & $(85,9)$ & \\
\hline Fundamental & 9 & $(11,5)$ & \\
\hline Renda per capita familiar ${ }^{a}$ & & & 0,923 \\
\hline Até 10 & 4 & $(5,1)$ & \\
\hline Até 5 & 15 & $(19,2)$ & \\
\hline Até 2 & 59 & $(75,6)$ & \\
\hline Situação conjugal & & & 0,628 \\
\hline Casado ou união estável & 3 & $(3,8)$ & \\
\hline Solteiro (nunca casou) & 74 & $(94,9)$ & \\
\hline Viúvo & 1 & $(1,3)$ & \\
\hline \multicolumn{4}{|l|}{ Estilo de vida } \\
\hline Fumo atual & & & 0,720 \\
\hline Não & 71 & $(91,0)$ & \\
\hline Sim & 7 & $(9,0)$ & \\
\hline Consumo de álcool & & & 0,930 \\
\hline Não & 36 & $(46,2)$ & \\
\hline Sim & 42 & $(53,8)$ & \\
\hline \multicolumn{4}{|l|}{ Distúrbios do sono } \\
\hline Dificuldade para dormir & & & 0,043 \\
\hline Não & 64 & $(82,1)$ & \\
\hline Sim & 14 & $(17,9)$ & \\
\hline $\begin{array}{l}\text { Acordar no meio da noite e } \\
\text { ter dificuldade para voltar a } \\
\text { adormecer }\end{array}$ & & & 0,019 \\
\hline Não & 65 & 83,3 & \\
\hline Sim & 13 & 16,7 & \\
\hline Estado nutricional $^{\mathrm{b}}$ & & & 0,067 \\
\hline Obesidade & 1 & $(1,3)$ & \\
\hline Sobrepeso & 13 & $(16,7)$ & \\
\hline Peso normal & 50 & $(64,1)$ & \\
\hline Abaixo do peso & 1 & $(1,3)$ & \\
\hline Desconhecido & 13 & $(16,7)$ & \\
\hline Aptidão cardiorrespiratória ${ }^{c}$ & & & 0,752 \\
\hline Superior & 31 & $(39,7)$ & \\
\hline Excelente & 22 & $(28,2)$ & \\
\hline Boa & 19 & $(24,4)$ & \\
\hline Regular & 3 & $(3,8)$ & \\
\hline Fraca & 3 & $(3,8)$ & \\
\hline Muito fraca & 0 & $(0,0)$ & \\
\hline
\end{tabular}

a Em salários mínimos; ${ }^{\mathrm{b}}$ Avaliado pelo Índice de Massa Corporal (IMC) e classificado segundo o Ministério da Saúde ${ }^{17}$; ${ }^{\mathrm{C}}$ Classificação de acordo com a metodologia de Cooper $^{20}$ 
Tabela 2. Razões de prevalência de transtornos mentais comuns segundo distúrbios do sono $(\mathrm{N}=$ 78), 2010

\begin{tabular}{lcc}
\hline \multicolumn{1}{c}{ Característica } & RP $^{\mathbf{a}}$ & $($ IC95\%) \\
\hline Dificuldade para adormecer & & \\
$\quad$ Não $^{\mathrm{b}}$ & - & \\
Sim & 4,17 & $(1,18-14,77)$ \\
Acordar no meio do sono e & & \\
ter dificuldade para voltar a & & \\
dormir & - & \\
$\quad$ Não & 5,69 & $(1,43-22,75)$ \\
Sim &
\end{tabular}

Razões de prevalência de transtornos mentais comuns (TMC), segundo distúrbios do sono, entre recrutas incorporados em 2010. ${ }^{a}$ RP: razões de prevalência; ${ }^{\text {b }}$ Categoria de referência

terísticas conferiu uma maior precisão das análises, em relação aos diversos fatores sob investigação, o que pode explicar a associação clara e única dos distúrbios do sono com TMC. Sobre esta associação, Sateia ${ }^{6}$ explica que a associação entre psicopatologia e sofrimento psíquico em distúrbios do sono é bi-direcional. Isto é, tanto transtornos mentais afetam a ocorrência de distúrbios do sono, quanto estes estão relacionados à ocorrência de transtornos mentais. No presente trabalho, partiu-se do pressuposto de que as mudanças na vida dos jovens recém-ingressos no EB causaram diversas alterações, sobretudo nas rotinas de sono, sendo plausível que a direção da associação seja de distúrbios do sono para TMC. No período inicial de incorporação, os recrutas passam por um processo de adaptação à vida militar, que inclui internato (dormir no quartel nas primeiras semanas), longas jornadas de treinamento, atividades de acampamentos para treinamentos militares e serviço de guarda ao quartel - ambas atividades caracterizadas por poucas e irregulares horas de sono. Como o EB tem, ao longo dos anos, diminuído o seu efetivo por restrições orçamentárias, muitas vezes a escala de serviço (quadro horário de quando o militar terá que estar de serviço de guarda ao quartel) apresenta-se muito apertada, podendo chegar às $24 \mathrm{~h}$ x 24h, ou seja, um dia de serviço para um dia de descanso. Todas estas circunstâncias podem restringir a quantidade e qualidade do sono dos jovens recrutas. Nesse sentido, um estudo longitudinal, em amostra de base populacional, conduzido na Austrália, com 20.820 jovens entre 17 e 24 anos, após um período de seguimento de 12-18 meses, demonstrou que há um aumento de trans- tornos mentais comuns entre aqueles que dormem pouco ${ }^{25}$.

Em relação aos transtornos mentais, a prevalência de TMC foi de 43,6\%, que se apresenta bem mais elevada do que a encontrada na população em geral $(28-35 \%)^{11,12}$ e entre jovens em idade semelhante, na cidade Pelotas - RS $(24,5 \%)^{26}$. Entretanto, pessoas que desempenham certas atividades profissionais tendem a apresentar prevalências maiores de transtornos mentais do que a população em geral, como é o caso, por exemplo, dos profissionais de saúde. Nesse sentido, nossos resultados se aproximaram bastante da prevalência encontrada por Braga et al. $^{27}(42,6 \%)$, sendo que a ocorrência de TMC, naqueles profissionais, estava associada às condições de trabalho.

Foi identificado um estudo, publicado recentemente, sobre saúde mental de militares das Forças Armadas do Brasil, que mostrou uma prevalência de transtornos mentais comuns, entre cabos e soldados de 33\%, sendo que o fenômeno não foi observado, com especificidade, nos soldados recrutas ${ }^{28}$, embora estes estivessem presentes no conjunto de dados. Nossos achados indicam que a prevalência de transtornos mentais comuns entre os recrutas parece ser maior do que em soldados mais antigos e cabos.

Comparando nossos resultados com estudo conduzido na Inglaterra, a prevalência encontrada de TMC foi de $23,5 \%$ entre cabos e soldados (other ranks) ${ }^{29}$. Como a presente investigação teve lugar no início do período do serviço militar obrigatório, pode-se hipotetizar que os recrutas estivessem passando por uma fase de adaptação às rotinas do quartel e, portanto, seria plausível que, passado este primeiro estágio, a prevalência de TMC entre os recrutas viesse a decair. Entretanto, esta hipótese só poderá ser confirmada por meio de estudo observacional de seguimento.

A prevalência de tabagismo entre os recrutas foi de $9 \%$, menor do que a de 15,5\% encontrada na população em geral de faixa etária e escolaridade semelhantes ${ }^{30}$. O consumo de álcool relatado pelos recrutas foi de 53,8\%, semelhantes aos dados de pesquisa nacional $(60,8 \%)^{31}$.

A população de estudo apresentou pouca variabilidade quanto à aptidão cardiorrespiratória, sendo que 92,3\% apresentaram níveis entre "Boa" e "Superior", de acordo com a escala de Cooper ${ }^{23}$. Devido à homogeneidade dos dados, não foi possível observar a variação da aptidão cardiorrespiratória em relação à variável dependente (TMC). Entretanto, é importante ressaltar que estudos têm mostrado que existe uma associação inversa en- 
tre aptidão cardiorrespiratória e transtornos mentais $^{32}$, ou seja, nível “Superior" de aptidão cardiorrespiratória está associado com menor risco de sintomas depressivos incidentes, independente de outros indicadores de risco clínico ${ }^{4}$.

Segundo a literatura, fatores como escolaridade e renda estão associados à ocorrência de $\mathrm{TMC}^{11}$, nesta população, provavelmente, devido à homogeneidade da amostra, também quanto a estas variáveis, não foi observada associação com TMC.

Observou-se que o estado nutricional apresentou uma significância estatística limítrofe ( $p$ $=0,067)$, entretanto, como cerca de $17 \%$ apresentavam dados ausentes (Tabela 1), optou-se por não incluir na análise multivariada a fim de se evitar distorções nos resultados.

\section{Pontos fortes e limitações do estudo}

Os jovens adultos (entre 18 e 19 anos) que, anualmente, se alistam no serviço militar obrigatório, passam por um processo de recrutamento, por ocasião da incorporação. Tal processo seleciona os mais saudáveis e que apresentam certas características socioeconômicas e demográficas. Sendo assim, a escolha da população proporcionou um controle prévio de algumas variáveis proporcionando uma diminuição de possível confundimento residual.

Dentre as limitações do estudo está sua natureza seccional, o que não permite avaliar causalidade nas relações entre os fenômenos observados.

Outra limitação do estudo é a impossibilidade de generalização dos resultados para todos os jovens adultos brasileiros entre 18-19 anos de idade, devido ao processo de recrutamento e seleção descrito anteriormente. Nessa perspectiva, nossos achados são aplicáveis somente a populações semelhantes.

\section{Considerações finais}

A prevalência de transtornos mentais comuns é elevada na população em geral, e os sintomas presentes nessa condição são tão ou mais incapacitantes do que quadros bem estabelecidos, segundo os códigos médicos ${ }^{8}$. Considerando-se o contexto em que as pesquisas, em países desenvolvidos, apontam que somente $40 \%$ das pessoas formalmente diagnosticadas com ansiedade e/ou depressão recebem o respectivo tratamento ${ }^{7}$, a urgência de mais pesquisas na área da saúde mental no Brasil fica ressaltada.

Até onde se sabe, este foi o primeiro estudo a investigar a saúde mental de jovens que prestam seu serviço militar obrigatório, sendo que a prevalência de transtornos mentais nos recrutas apresentou-se bem maior do que em jovens da população em geral, e do que em militares ingleses de postos semelhantes. Estes achados parecem indicar que as situações vivenciadas no ambiente de trabalho pelos recém-ingressos podem estar causalmente associadas à ocorrência de transtornos mentais comuns. Nessa perspectiva, sugere-se que outros estudos de desenho longitudinal sejam conduzidos entre os recrutas, para que sejam formuladas inferências causais sobre o tema e, a partir de tais conhecimentos, seja possível viabilizar o desenvolvimento de linhas de ação, tanto para o planejamento do treinamento militar, quanto para o atendimento em saúde mental oferecido aos jovens soldados do Exército Brasileiro, durante as diferentes fases do serviço militar obrigatório. 


\section{Colaboradores}

LCX Martins e L Kuhn participaram igualmente de todas as etapas de elaboração do artigo.

\section{Referências}

1. Organização Mundial da Saúde (OMS). Organização Panamericana de Saúde (OPAS). Relatório sobre a saúde no mundo 2001 - Saúde Mental: Nova Concepção, Nova Esperança. Washington: OPAS; 2001.

2. Lopes CS, Faerstein E, Chor D. Eventos de vida produtores de estresse e transtornos mentais comuns: resultados do Estudo Pró-Saúde. Cad Saude Publica 2003; 19(6):1713-1720.

3. Coutinho E, Almeida F, Mari J. Fatores de risco para morbidade psiquiátrica menor: resultados de um estudo transversal em três áreas urbanas no Brasil. Rev Psiq Clín 1999; 26(5):246-256.

4. Sui X, Laditka JN, Church TS, Hardin JW, Chase N, Davis K, Blair SN. Prospective study of cardiorespiratory fitness and depressive symptoms in women and men. J Psychiatr Res 2009; 43(5):546-552.

5. Kivimaki M, Jokela M, Hamer M, Geddes J, Ebmeier K, Kumari M, Singh-Manoux A, Hingorani A, Batty GD. Examining overweight and obesity as risk factors for common mental disorders using fat mass and obesity-associated (FTO) genotype-instrumented analysis: The Whitehall II Study, 19852004. Am J Epidemiol 2011;173(4):421-429.

6. Sateia MJ. Update on sleep and psychiatric disorders. Chest 2009;135(5):1370-1379.

7. Chopra P. Mental health and the workplace: issues for developing countries. Int J Ment Health Syst 2009; 3(1):4.

8. Maragno L, Goldbaum M, Gianini RJ, Novaes HMD, César CLG. Prevalência de transtornos mentais comuns em populações atendidas pelo Programa Saúde da Família (QUALIS) no Município de São Paulo, Brasil. Cad Saude Publica 2006; 22(8):1639-1648.

9. Wang PS, Simon G, Kessler RC. The economic burden of depression and the cost-effectiveness of treatment. Int J Methods Psychiatr Res 2003;12(1):22-33.

10. Goldberg D. The detection of psychiatric illness by questionnaire: London: OUP; 1972.

11. Ludermir AB. Desigualdades de classe e gênero e saúde mental nas cidades. Physis: Revista de Saúde Coletiva 2008; 18(3):451-467.

12. Anselmi L, Barros FC, Minten GC, Gigante DP, Horta BL, Victora CG. Prevalence and early determinants of common mental disorders in the 1982 birth cohort, Pelotas, Southern Brazil. Rev Saude Publica 2008; 42(Supl. 2):26-33.

13. Jones M, Rona RJ, Hooper R, Wesseley S. The burden of psychological symptoms in UK Armed Forces. Occup Med 2006; 56(5):322-328.

14. Hourani LL, Williams TV, Kress AM. Stress, mental health, and job performance among active duty military personnel: findings from the 2002 Department of Defense Health-Related Behaviors Survey. Mil Med 2006; 171(9):849-856.

15. Souza WF, Figueira I, Mendlowicz MV, Volchan E, Mendonca-de-Souza AC, Duarte AF, Monteiro da Silva AM, Marques-Portella C, Mari JJ, Coutinho ES. Negative affect predicts posttraumatic stress symptoms in Brazilian volunteer United Nations peacekeepers in Haiti. J Nerv Ment Dis 2008; 196(11): 852-855. 
16. Kuhlman PRL. Serviço militar obrigatório no Brasil: continuidade ou mudança? Security and Defense Studies Review 2001; 1:147-158.

17. Goldberg D, Williams P. A user's guide to the General Health Questionnaire. Windsor: Nelson Publishing; 1988.

18. Gouveia VV, Chaves SSS, Oliveira ICP, Dias MR, Gouveia RSV, Andrade PR. A utilização do QSG-12 na população geral: estudo de sua validade de construto. Psicologia: Teoria e Pesquisa 2003; 19(3):241-248.

19. Mari JJ, Williams P. A comparison of the validity of two psychiatric screening questionnaires (GHQ-12 and SRQ-20) in Brazil, using Relative Operating Characteristic (ROC) analysis. Psychol Med 1985; 15(3):651-659.

20. Brasil, Ministério da Saúde (MS). Vigilância alimentar e nutricional - SISVAN: Orientações básicas para a coleta, o processamento, análise de dados e a informação em serviços de saúde. Brasília: MS; 2004.

21. Franchini E, Nunes AV, Moraes JM, Del Vecchio FB. Physical fitness and anthropometrical profile of the Brazilian male judo team. J Physiol Anthropol 2007; 26(2):59-67.

22. McArdle WD, Katch FI, Katch VL. Exercise physiology: Energy, nutrition and human performance. $5^{\text {t }}$ Edition. Norristown: Lippincott Williams \& Wilkins; 2007.

23. Cooper KH. Physical training programs for mass scale use: effects on cardiovascular disease - facts and theories. Ann Clin Res 1982; 14(Supl. 34):25-32.

24. R Development Core Team R. R: A language and environment for statistical computing. Vienna: R Foundation for Statistical Computing; 2009.

25. Glozier N, Martiniuk A, Patton G, Ivers R, Li Q, Hickie I, Senserrick T, Woodward M, Norton R, Stevenson M. Short sleep duration in prevalent and persistent psychological distress in young adults: the DRIVE study. Sleep 2010; 33(9):1139-1145.
26. Jansen K, Mondin TC, Ores LdC, Souza LDdM, Konradt CE, Pinheiro RT, Silva RA. Transtornos mentais comuns e qualidade de vida em jovens: uma amostra populacional de Pelotas, Rio Grande do Sul, Brasil. Cad Saude Publica 2011; 27(3):440-448.

27. Braga LC, Carvalho LR, Binder MCP. Condições de trabalho e transtornos mentais comuns em trabalhadores da rede básica de saúde de Botucatu (SP). Ciên Saude Colet 2010; 15(Supl.):1585-1596.

28. Martins LCX, Lopes CS. Military hierarchy, job stress and mental health in peacetime. Occupational Medicine 2012; 62(3):182-187.

29. Fear NT, Rubin GJ, Hatch S, Hull L, Jones M, Hotopf M, Wessely S, Rona RJ. Job strain, rank, and mental health in the UK Armed Forces. Int J Occup Environ Health 2009; 15(3):291-298.

30. Silva GA, Valente JG, Almeida LMd, Moura ECd, Malta DC. Tabagismo e escolaridade no Brasil, 2006. Rev Saude Publica 2009; 43(Supl. 2):48-56.

31. Brasil. Ministério da Saúde (MS). Instituto Nacional do Câncer (Inca). Inquérito Domiciliar sobre Comportamentos de Risco e Morbidade Referida de Doenças e Agravos não Transmissíveis. Brasília: Inca; 2003

32. Roth DL, Holmes DS. Influence of physical fitness in determining the impact of stressful life events on physical and psychologic health. Psychosom Med 1985; 47(2):164-173.

Artigo apresentado em 04/03/2012

Aprovado em 28/03/2012

Versão final apresentada em 03/04/2012 